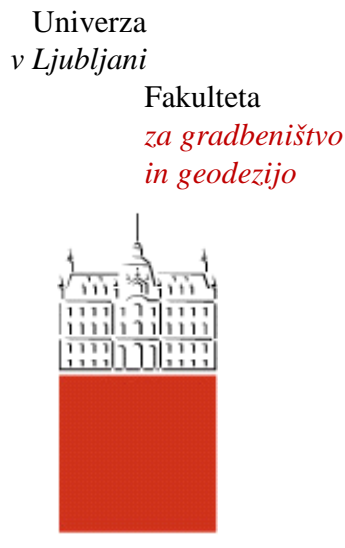

Jamova 2

1000 Ljubljana, Slovenija http://www3.fgg.uni-lj.si/

DRUGG - Digitalni repozitorij UL FGG http://drugg.fgg.uni-lj.si/

Ta članek je avtorjeva zadnja recenzirana različica, kot je bila sprejeta po opravljeni recenziji.

Prosimo, da se pri navajanju sklicujete na bibliografske podatke, kot je navedeno:

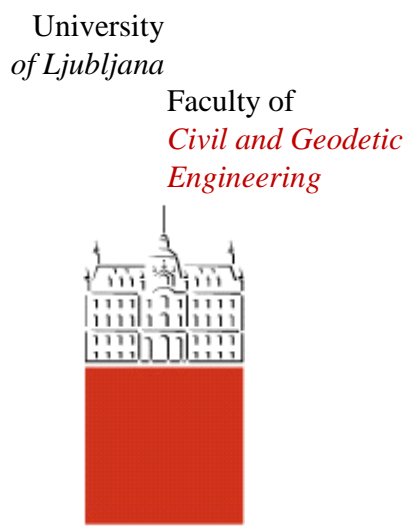

Jamova 2

SI - 1000 Ljubljana, Slovenia http://www3.fgg.uni-lj.si/en/

DRUGG - The Digital Repository http://drugg.fgg.uni-lj.si/

This version of the article is author's manuscript as accepted for publishing after the review process.

When citing, please refer to the publisher's bibliographic information as follows:

Turk, G. in Ranta-Maunus A. 2004. Analysis of strength grading of sawn timber based on numerical simulation. Wood Science and Technology 38, 7: 493-505.

DOI: 10.1007/s00226-004-0254-4. 


\title{
Analysis of strength grading of sawn timber based on numerical simulation
}

\author{
GORAN TURK ${ }^{1}$, AlPO RANTA-MAUNUs ${ }^{2}$ \\ ${ }^{1}$ University of Ljubljana, Faculty of Civil and Geodetic Engineering, Jamova 2, \\ 1000 Ljubljana, Slovenia \\ gturk@.fgg.uni-lj.si \\ phone: +38614768614 \\ fax: $\quad+38614768629$ \\ ${ }^{2}$ VTT, Technical Research Centre of Finland, P.O.Box 1806, FIN-02044 VTT, \\ Finland \\ Alpo.Ranta-Maunus@vtt.fi \\ phone +35894565536 \\ fax $\quad+35894567027$
}

\begin{abstract}
The main problem in machine strength grading of wood lies in the grade determining parameters and machine grading parameters, which are all stochastic, and the correlation between them is of critical importance. Therefore it is difficult to reliably determine the grade of a particular piece. In order to understand the effectiveness and accuracy of machine grading one should perform a large number of experiments in which the non-destructive machine grading parameters as well as destructive grade determining parameters should be determined. So far we have had access to only limited number of such experiments. The basic idea of this paper is to use experimental data only for the determination of statistical parameters - mean vector and variance- covariance matrix and afterwards use these statistical parameters in numerical simulation of machine grading. This numerical procedure gave some valuable data on the average values and the variability of grading results. As a result it is now easier to decide what sample size one should use in determining machine settings.
\end{abstract}

Keywords: timber, machine grading, strength grading, numerical simulations, random number generation 


\section{Introduction}

Machine strength grading of sawn timber is based on correlations between grade determining properties like strength, stiffness and density, and measured physical properties. When new types of machines are taken into use, they are calibrated with timber sample representing the wood material for grading of which the machine will be used. Correlations between grade determining and measured properties depend on growth conditions, and this is taken into account by sampling the specimens from different growth regions.

Another aspect is the statistical nature of timber strength: there is no method which could exactly predict the strength of a certain piece of wood. However, there are known correlations between strength and measurable physical properties. The consequence is that thousands of specimens need to be tested to establish settings for grading machines, and after having done it, there is still an uncertainty about the strength distribution of produced strength graded timber as well as about the yield of timber to different grades.

This paper analyses the statistical effects in the ideal situation when sampling has been done perfectly and all sub-samples belong to same population with normally distributed properties. We concern grading of timber, mainly taking into account the forthcoming European standard (prEN 14081-2, 2002). Based on earlier experiments, correlations between grade determining properties, strength, modulus of elasticity and density will be assumed, and the numerical simulation of the standard grading procedure will be applied to larger populations than can be afforded in testing. This approach was illustrated in an earlier paper (RantaMaunus, 2002). Now we use a more advanced statistical procedure for the simulation of wood characteristics.

This method is expected to be useful in the analysis of issues, such as:

- sensitivity of grading result to the initial distributions of properties and to the effectiveness of the grading method

- $\quad$ sensitivity of grading result to sample size and to statistical methods used when settings of machine are determined

- yield to different grades when graded simultaneously to one or more grades

- form of lower tail of strength distribution of different grades. 
In order to reliably estimate the accuracy of machine grading, the sampling was repeated several times. Thus, the average values of yield in different grades and other parameters describing the characteristics of graded timber were evaluated. It was assumed that all parameters of ungraded population of timber are jointly normally distributed. This assumption was verified by statistical analysis of the population of actual experimental results.

\section{Numerical generation of sample}

It is assumed that grade determining parameters: strength $f$, modulus of elasticity $E$ and density $\rho$, and the observed grading parameter $E_{\text {mach }}$ are all normally distributed random variables. The characteristics of this random vector are presented by its mean value vector $\mathbf{m}_{\mathbf{Y}}$ and its variance- covariance matrix $\boldsymbol{\Sigma}_{\mathbf{Y}}$

$$
\begin{aligned}
& \mathbf{m}_{\mathbf{Y}}=E[\mathbf{Y}]=\left[\begin{array}{l}
m_{Y_{1}} \\
m_{Y_{2}} \\
m_{Y_{3}} \\
m_{Y_{4}}
\end{array}\right] \\
& \boldsymbol{\Sigma}_{\mathbf{Y}}=E\left[\left(\mathbf{y}-\mathbf{m}_{\mathbf{Y}}\right)\left(\mathbf{y}-\mathbf{m}_{\mathbf{Y}}\right)^{T}\right]=\left[\begin{array}{cccc}
\sigma_{Y_{1}}^{2} & \sigma_{Y_{1}, Y_{2}} & \sigma_{Y_{1}, Y_{3}} & \sigma_{Y_{1}, Y_{4}} \\
\sigma_{Y_{1}, Y_{2}} & \sigma_{Y_{2}}^{2} & \sigma_{Y_{2}, Y_{3}} & \sigma_{Y_{2}, Y_{4}} \\
\sigma_{Y_{1}, Y_{3}} & \sigma_{Y_{2}, Y_{3}} & \sigma_{Y_{3}}^{2} & \sigma_{Y_{3}, Y_{4}} \\
\sigma_{Y_{1}, Y_{4}} & \sigma_{Y_{2}, Y_{4}} & \sigma_{Y_{3}, Y_{4}} & \sigma_{Y_{4}}^{2}
\end{array}\right]
\end{aligned}
$$

The mean value vector as well as variance- covariance matrix are estimated from a sample of real experimental results $y_{i k}, i=1, \ldots, 4, k=1, \ldots, n$, where $n$ is the sample size.

\section{Generating a sample of normally distributed random vector}

The basic idea behind generating a sample of dependent normally distributed random variables is to generate a sample of independent normally distributed random variables and then use a linear transformation to obtain a sample of dependent random variables (e.g. Devroy 1986).

\section{Linear transformation}

The linear transformation of random vector $\mathbf{X}$, which is taken to be a set of independent random variables with zero mean and unit variances, is defined as 


$$
\mathbf{Y}=\mathbf{H} \mathbf{X}
$$

where $\mathbf{H}$ is transformation matrix, which deforms the coordinate system.

Very important linear transformations are rotations which correspond to

orthonormal transformation matrix $\mathbf{H}$.

The variance-covariance matrix of random vector $\mathbf{X}$ is an identity matrix.

\section{Generating a random vector with a given variance-covariance matrix}

Let us assume that random vector $\mathbf{Y}$ has zero mean values. In this case the variance-covariance matrix is defined as follows

$$
\boldsymbol{\Sigma}=E\left[\mathbf{Y} \mathbf{Y}^{T}\right]
$$

where $E[$.$] denotes the expected values. If we use Equ. (4) in Equ. (5), we obtain a$ useful relationship

$$
\boldsymbol{\Sigma}=E\left[\mathbf{Y} \mathbf{Y}^{T}\right]=E\left[\mathbf{H} \mathbf{X} \mathbf{X}^{T} \mathbf{H}^{T}\right]=\mathbf{H} E\left[\mathbf{X} \mathbf{X}^{T}\right] \mathbf{H}^{T},
$$

Since the variance-covariance matrix of $\mathbf{X}$ is identity matrix $E\left[\mathbf{X} \mathbf{X}^{T}\right]=\mathbf{I}$, Equ.

(6) reduces to

$$
\boldsymbol{\Sigma}=\mathbf{H} \mathbf{H}^{T},
$$

We have to find such matrix $\mathbf{H}$, for which Equ. (7) holds. Since the variance-covariance matrix is non-singular symmetric matrix, the problem can easily be solved by Cholesky decomposition (see e.g. Press et al. 1992).

\section{Generating procedure}

The generation of normally distributed random vector with known variance-covariance matrix is carried out by the following procedure:

1. Perform a Cholesky decomposition of variance-covariance matrix $\boldsymbol{\Sigma}$ of random vector $\mathbf{X}$ (determination of $\mathbf{H}$ ).

2. Generate a set of independent random variables with standardized normal distribution (generation of random vector $\mathbf{X}$ ).

3. Perform a transformation which transforms independent variables $\mathbf{X}$ into random vector $\mathbf{Y}$ with known variance-covariance matrix (use of equation $\mathbf{Y}=\mathbf{H} \mathbf{X})$. 
4. Perform an additional transformation to obtain a random vector $\mathbf{Z}$ with nonzero mean values (use of equation $\mathbf{Z}=\mathbf{Y}+E[\mathbf{Z}]$ ).

\section{Optimum grading}

When settings of a grading machine are determined according to EN 14081, both non-destructive testing by the use of grading machine, and destructive testing for the determination of the real grade determining properties are made for a representative sample. The first step in the analysis of the results is the determination of the "real" grade of each specimen based on the information of destructive testing: bending strength, modulus of elasticity and density. This grading is made in such way that each specimen would be placed in as high grade as possible, and it is therefore called optimum grading. In our case a simultaneous grading to three grades was performed: $\mathrm{C} 40, \mathrm{C} 30$ and $\mathrm{C} 18$. The modified requirements for these grades according to prEN 14081 [1] are shown in Tab. 1.

\section{Table 1}

During the optimum grading determination we would like to grade as many pieces as possible to higher grades, with the following requirements (constraints):

1. the sample (grade) $5 \%$ percentile of the strength $f_{0.05}$ is higher than $f_{r}$,

2. the sample (grade) mean (average) of $\bar{E}$ is higher than $E_{r}$,

3. the sample (grade) $5 \%$ percentile of the density $\rho_{0.05}$ is higher than $\rho_{r}$,

4. the sample $5 \%$ percentile of the strength $f_{0.05}^{a}$ is higher than $f_{r}$ for the subsample obtained just by ranking according to the strength,

5. the sample mean of $\bar{E}^{a}$ is higher than $E_{r}$ for the sub-sample obtained just by grading according to the modulus of elasticity, and

6. the sample $5 \%$ percentile of the density $\rho_{0.05}^{a}$ is higher than $\rho_{r}$ for the subsample obtained just by ranking according to the density.

A computer code (written in MATHEMATICA) was prepared for automatic determination of optimum grading. In addition to these requirements (constraints) the objective function $S$ was defined as

$$
S=\frac{\left|f_{0.05}-f_{r}\right|}{f_{r}}+\frac{\left|\bar{E}-E_{r}\right|}{E_{r}}+\frac{\left|\rho_{0.05}-\rho_{r}\right|}{\rho_{r}}+\frac{\left|f_{0.05}^{a}-f_{r}\right|}{f_{r}}+\frac{\left|\bar{E}^{a}-E_{r}\right|}{E_{r}}+\frac{\left|\rho_{0.05}^{a}-\rho_{r}\right|}{\rho_{r}}
$$


It is our goal to find such settings of limits used in optimum grade determination that function $S$ reaches its minimum. This set of limits gives the optimum grading of the sample or population.

The optimization procedure itself utilizes a relatively simple step-by-step bisectionlike method, in which the limits for any of grading determining parameters are decreased by a certain step-size if the difference (e.g. $f_{0.05}-f_{r}$ ) is positive and increased if it is negative. The increasing is four times faster than the decreasing. When all three limits (for $f, E$ and $\rho$ ) are as low as possible with the conditions fulfilled, the step-size is halved and the procedure is repeated. This simple method, which emulates the manual determination of optimal grading, gives very accurate limits for the grade determining parameters. Thus, the obtained grading is almost certainly the optimal one.

\section{Characteristic value determination}

The characteristic value has been determined by the use of order statistics. E.g., let us determine the $5 \%$ percentile $y_{k}$ from the sample $y_{i}=1, \ldots, n$ where $n$ is sample size. The sample is sorted so that $y_{i} \leq y_{i+1}$. The sample element $y_{j}$ which satisfies the condition

$$
j=\lfloor 0.05 n\rfloor,
$$

is the highest among the elements which are below the characteristic value. The notation $j=\lfloor x\rfloor$ denotes the floor of the number $x$, i.e. the greatest integer less than or equal to $x$. The characteristic value is finally determined by the equation

$$
y_{k}=y_{j}+(0.05 n-j)\left(y_{j+1}-y_{j}\right) \text {, }
$$

which represents the linear interpolation between the two values embracing the characteristic value. If the sample size is lower than $1 / 0.05=20$ the equation (10) cannot be used. It is in this case difficult to reliably determine the characteristic value. An approximate formula can be used

$$
y_{k}=0.05 n y_{1} \text {. }
$$




\section{Machine grading}

Machine grading is carried out according to the machine settings determined by comparison of machine readings and real grade determining parameters. Here, a procedure is introduced for automatic determination of settings according to prEN 14081-2. The three limits for grades C40, C30 and C18 will be set in such way that the following conditions are fulfilled:

1. the sample (grade) $5 \%$ percentile of the strength $f_{0.05}$ is higher than $f_{r}$,

2. the sample (grade) mean (average) of $\bar{E}$ is higher than $E_{r}$,

3. the sample (grade) $5 \%$ percentile of the density $\rho_{0.05}$ is higher than $\rho_{r}$,

4. none of the cells in the global cost matrix which indicate wrongly upgraded are grater than 0.2 ,

5. the number of rejected pieces is grater or equal to $0.5 \%$ of the total number of pieces in the sample.

Similarly as for optimum grading determination, an automatic optimization procedure was developed in determining machine settings. There are several options for the objective function: different objective functions can be defined from global cost matrix or based on the number of pieces assigned to a particular grade. In our analysis objective function is a weighted sum of the number of pieces assigned to all grades:

$$
S=9 n_{c 40}+3 n_{c 30}+n_{c 18} .
$$

We start from random choices of settings, and among the cases that fulfil the requirements the one with the highest objective function is chosen. From that point the limits of machine settings were lowered as much as possible so that the requirements are fulfilled and the objective function is maximized. This procedure usually gives relatively high yields in higher grades.

\section{Numerical example}

Based on the available data of 589 pieces of spruce with the depth of $150 \mathrm{~mm}$, graded by a traditional bending type machine (Ranta-Maunus et al. 2001), the 
descriptive statistics were determined. Descriptive statistics may be summarized in mean vector $\mathbf{m}$ and variance- covariance matrix $\boldsymbol{\Sigma}$

$$
\mathbf{m}=\left[\begin{array}{c}
45.247 \\
13003.17 \\
448.00 \\
9495.9
\end{array}\right] \quad \boldsymbol{\Sigma}=\left[\begin{array}{cccc}
129.906 & 21759.6 & 263.13 & 10740.7 \\
21759.6 & 5.98507 \cdot 10^{6} & 66481.3 & 2.47506 \cdot 10^{6} \\
263.13 & 66481.3 & 1630.76 & 37881.2 \\
10740.7 & 2.47506 \cdot 10^{6} & 37881.2 & 1.48582 \cdot 10^{6}
\end{array}\right]
$$

Correlations between these variables are more evident in correlation matrices $\mathbf{R}$ :

$$
\mathbf{R}=\left[\begin{array}{cccc}
1 & 0.78037 & 0.57169 & 0.77310 \\
0.78037 & 1 & 0.67293 & 0.82998 \\
0.57169 & 0.67293 & 1 & 0.76957 \\
0.77310 & 0.82998 & 0.76957 & 1
\end{array}\right]
$$

Different sample sizes for machine settings were chosen: 125, 250, 500, 750, 1000, 1250 and 2500 . The determination of machine settings was repeated 100 times with independent samples.

The results for mean machine setting parameters are shown in Tab. 2. The average values of machine settings do not differ much for different sample sizes from 250 to 2500 pieces. In the case of the smallest sample of 125 pieces, there is a considerable probability (approx. $40 \%$ ) that would be impossible to grade any piece to the highest grade $\mathrm{C} 40$. Therefore, we conclude that the grading to the three grades simultaneously based on the smallest sample of 125 pieces is not adequately appropriate and is omitted in further analysis.

\section{Table 2}

The values of grading settings obtained by 100 repetitions were applied on the same population of 10000 pieces. The results are shown in Tab. 3.

\section{Table 3}

With this calculation the yields in individual classes are determined. The ratio between $5 \%$ and $0.5 \%$ percentile of strength is determined for all three classes. 
In case there were not enough pieces in a particular grade, $0.5 \%$ percentile has not been determined.

The yield in C40 was approx. $36 \%$ and in C30 between 50 and $55 \%$ (compared to optimum grade yield of $62 \%$ and $29 \%$ in C40 and C30, respectively). The results improve slightly if the sample size is increased from 250 to 2500 (see Fig. $1)$.

\section{Figure 1}

There is an evident improvement in COV of yield which decreases from above 0.25 in the case of $n=250$ to below 0.1 in the case of $n=1250$ (see Fig. 2). This reduction in COV results in much narrower confidence interval for the cases when the machine grading was determined from only a few samples.

\section{Figure 2}

Other parameters do not change for different sample sizes, e.g. the ratio $f_{0.005} / f_{0.05}$ is virtually constant for all sample sizes.

\section{The influence of dependent sampling}

In real determination of machine settings we will not be able to have a large number of independent samples. In fact, we may have only a relatively large population (e.g. $N=1000$ ) of all available measurements from which we may draw several samples. These samples are obviously not independent, since the population from which we take samples is not infinite.

In this numerical example we illustrate the effect of dependent sampling, i.e. sampling from a finite population. First we generate the population of 1000 pieces ( $N=1000$ ). Then we randomly select samples from this population. In this case it does not make any sense to analyse larger samples, thus only samples of size 125 , 250,500 and 750 were analysed.

We may see that the effect from sampling from the population of 1000 is not very evident. We can see a slight reduction in variance which is presented in Tab. 4 and clearly illustrated in Fig. 3.

\section{Figure 3}

\section{Table 4}


However, it has to be emphasized that the result depends on the random sample of 1000 pieces which in this case presents population. From Tab. 2 and 3 we can see that repeating of samples of 1000 pieces does not give equal results; e.g. COV of machine grading parameters are between 1.6 and $6.6 \%$ for different grades and COV of yield is between 13.5 and $57.8 \%$. Thus, we can expect that if different population of 1000 is used in the analysis, one would get different results. Numerical results which confirm this expectation are shown in Tab. 5. Here the dependent sampling from three populations of 1000 was repeated 100 times. We can see that averages as well as standard deviations of machine settings depend on the particular population of 1000 . The differences are much more evident than those observed in Tab. 4, where dependent sampling for different sample sizes were repeated 100 times but the same population of 1000 was used.

\section{Table 5}

\section{Discussion}

In the numerical example we used prEN 14081-2 approach with the following deviation: 100 independent samples were generated separately for sample sizes from 125 to 2500, and used for the determination of settings, whereas in standard procedure much smaller number of dependent samples is used.

First we analyse how the yield to different grades is influenced by the random factors, and how they can be counteracted by increasing the sample size.

We observe that when the sample size is increased, the yield to higher grades increases slightly, whereas COV of yield decreases considerably (see Tab. 3 and Fig. 1 and 2). The results which are summarised in Tab. 6 and illustrated in Fig. 4 indicate that when settings are determined by the use of "standard" sampling (4 samples of 250 pieces), the machine gives on average $35.3 \%$ yield to $\mathrm{C} 40$, but it may give a yield from 26.2 to $44.4 \%$ within confidence limits of $95 \%$. When four times larger sample is used for determining the settings, we obtain mean yield $37.1 \%$ and confidence limits are from 32.2 to $42.0 \%$.

\section{Figure 4}

\section{Table 6}


Also the form of strength distribution of different grades was studied. Based on the data from $150 \mathrm{~mm}$ wide spruce as above, we analysed the strength distributions of 10000 specimens when graded simultaneously to C40, C30 and C18 with settings based on mean values of 100 simulations of independent samples. We learned that the result is quite independent of the sample size, the average ratios between $0.5 \%$ and $5 \%$ percentile of the strength are $0.828,0.753$, and 0.605 in classes $\mathrm{C} 40, \mathrm{C} 30$, and $\mathrm{C} 18$, respectively. The variance of the ratio is in generally lower if larger samples were taken into account (see Fig. 5). The form of strength distribution in lower tail area is illustrated in Fig. 6, where the $5 \%$ percentile strength of each grade is denoted by 1 and cumulative distribution of the relative strength is shown. The curves are lognormal distributions which are fitted to simulated results in two points: $f_{0.005}$ and $f_{0.05}$. COV of the fitted lognormal distribution is indicated in Fig. 6, 0.2 for $\mathrm{C} 40$ and 0.6 for $\mathrm{C} 18$. We observed that $\mathrm{C} 40$ is generally much better material than lower grades.

\section{Figure 5 \\ Figure 6}

We have to keep in mind that this analysis is based on purely statistical effects and represents therefore an ideal case. When the growth region and range of sizes where the same strength model is used, are maximised, the effectiveness of grading is lower, and our numerical results are not directly valid. The power of numerical simulation is, however, in number of simulations: we can study statistical phenomena with large sample sizes which cannot be afforded in destructive testing.

\section{Conclusions}

It was important to use actual experimental data to determine random vector characteristics (mean and variance- covariance matrix) used in sample generation. The data for spruce pieces with the depth of $150 \mathrm{~mm}$ showed quite high correlation between grade determining parameters and grading parameters. As a result the machine grading proved to be very successful. It is shown that the yield to higher grades increased noticeably with larger sample sizes. Even more important is the variance reduction for all the studied parameters: yield and percentile ratio for all grades. Numerical results indicate that the form of lower tail of strength 
distribution in high grades is different from low grades: portion of specimens having strength considerably below the acceptance limit is less for $\mathrm{C} 40$ than for C18.

Sampling from finite size population results in reducing the variances in machine settings, but this reduction is not due to better procedure but due to dependent sampling, which may lead to biased results.

\section{References}

prEN 14081-2 (2992) Timber structures - Strength graded structural timber with rectangular cross section Part 2: Machine Grading - Additional requirements for initial type testing, European Committee for Standardization, Brussels

Devroy L (1986) Non-Uniform Random Variate Generation, Springer-Verlag: New York

Press WH, Teukolsky SA, Vetterling WT, Flannery BP (1992) Numerical Recipes in Fortran, The Art of Scientific Computing, Second Edition, Cambridge University Press, Cambridge (UK)

Wolfram S (1991) Mathematica, A System for Doing Mathematics by Computer, Second Edition, AddisonWesley Publishing Company, Inc., Redwood City (Ca)

Ranta-Maunus A (2002) The effect of machine strength grading on the strength distribution of sawn timber based on numerical simulation. COST Action E24 \& JCSS Workshop in Zürich, October 10-11, 2002

Ranta-Maunus A, Fonselius M, Kurkela J, Toratti T (2001) Reliability analysis of timber structures. VTT Research Notes 2109. Espoo, Finland 
Figure 1: Average yield in C40 and C30 for the generated samples of spruce with $150 \mathrm{~mm}$ depth

Figure 2: Coefficient of variation for yield in $\mathrm{C} 40$ and $\mathrm{C} 30$ for the generated samples of spruce with $150 \mathrm{~mm}$ depth

Figure 3: Coefficients of variation of machine settings for the cases of independent sampling and sampling from the finite population of 1000 pieces

Figure 4: Yield in C40 and C30 - 95\% confidence intervals (4 repetitions of sampling)

Figure 5: Ratio between $0.5 \%$ and $5 \%$ percentile of the strength

Figure 6: Relative strength of timber graded simultaneously to C40, C30 and C18 
Table 1: Modified requirements for grades C40, C30 and C18

\begin{tabular}{|l|c|c|c|}
\hline Grade & $\begin{array}{c}f_{r} \\
{\left[\mathrm{~N} / \mathrm{mm}^{2}\right]}\end{array}$ & $\begin{array}{c}E_{r} \\
{\left[\mathrm{~N} / \mathrm{mm}^{2}\right]}\end{array}$ & $\begin{array}{c}\rho_{r} \\
{\left[\mathrm{~kg} / \mathrm{m}^{3}\right]}\end{array}$ \\
\hline C40 & 40.0 & 13300 & 420 \\
\hline C30 & 26.8 & 11400 & 380 \\
\hline C18 & 16.1 & 8550 & 320 \\
\hline
\end{tabular}


Tab. 2: Mean machine settings for different sample sizes

\begin{tabular}{|c|l|c|c|c|}
\hline \multirow{2}{*}{$\begin{array}{l}\text { Sample } \\
\text { size }\end{array}$} & & \multicolumn{3}{|c|}{ Grades } \\
\cline { 3 - 5 } & & $\mathbf{C 4 0}$ & $\mathbf{C 3 0}$ & $\mathbf{C 1 8}$ \\
\hline \multirow{2}{*}{250} & average & 9944.4 & 8211.6 & 6767.4 \\
\cline { 2 - 5 } & st. deviation & 316.6 & 403.8 & 593.5 \\
\hline \multirow{2}{*}{500} & average & 9904.8 & 7886.7 & 6478.9 \\
\cline { 2 - 5 } & st. deviation & 205.5 & 260.2 & 263.1 \\
\hline \multirow{2}{*}{750} & average & 9884.9 & 7821.0 & 6467.9 \\
\cline { 2 - 5 } & st. deviation & 169.2 & 224.1 & 299.4 \\
\hline \multirow{2}{*}{1000} & average & 9855.8 & 7754.5 & 6589.9 \\
\cline { 2 - 5 } & st. deviation & 137.2 & 207.5 & 270.3 \\
\hline \multirow{2}{*}{1250} & average & 9892.1 & 7734.9 & 6463.0 \\
\cline { 2 - 5 } & st. deviation & 115.5 & 194.2 & 235.9 \\
\hline \multirow{2}{*}{2500} & average & 9857.6 & 7688.1 & 6478.6 \\
\cline { 2 - 5 } & st. deviation & 96.0 & 140.4 & 210.6 \\
\hline
\end{tabular}


Table 3: Grading results for the population of 10000 pieces, machine settings determined from samples of different sizes, samplings were repeated 100 times.

\begin{tabular}{|c|c|c|c|c|c|c|c|}
\hline & \multicolumn{6}{|c|}{ Grades } \\
\hline & & \multicolumn{2}{|c|}{$\mathrm{C40}$} & \multicolumn{2}{|c|}{ C30 } & \multicolumn{2}{|c|}{$\mathrm{C} 18$} \\
\hline & & $\begin{array}{c}\text { Yield } \\
{[\%]}\end{array}$ & $\frac{f_{0.005}}{f_{0.05}}$ & $\begin{array}{c}\text { Yield } \\
{[\%]}\end{array}$ & $\frac{f_{0.005}}{f_{0.05}}$ & $\begin{array}{c}\text { Yield } \\
{[\%]}\end{array}$ & $\frac{f_{0.005}}{f_{0.05}}$ \\
\hline $\begin{array}{l}\text { Sample } \\
\text { size }\end{array}$ & $\begin{array}{l}\text { Optimum } \\
\text { grading }\end{array}$ & 61.9 & 0.971 & 28.7 & 0.916 & 9.4 & 0.434 \\
\hline \multirow{2}{*}{250} & average & 35.3 & 0.828 & 48.6 & 0.768 & 13.6 & 0.624 \\
\hline & st. deviation & 9.3 & 0.0104 & 13.9 & 0.0179 & 7.9 & 0.0602 \\
\hline \multirow{2}{*}{500} & average & 35.9 & 0.828 & 50.8 & 0.763 & 11.0 & 0.616 \\
\hline & st. deviation & 6.1 & 0.0075 & 9.6 & 0.0188 & 6.4 & 0.0624 \\
\hline \multirow{2}{*}{750} & average & 36.7 & 0.828 & 54.4 & 0.748 & 8.1 & 0.603 \\
\hline & st. deviation & 5.4 & 0.0061 & 6.9 & 0.0170 & 3.1 & 0.0227 \\
\hline \multirow{2}{*}{1000} & average & 37.6 & 0.827 & 54.4 & 0.743 & 7.0 & 0.583 \\
\hline & st. deviation & 4.4 & 0.0057 & 5.7 & 0.0161 & 2.7 & 0.0733 \\
\hline \multirow{2}{*}{1250} & average & 36.4 & 0.828 & 55.9 & 0.743 & 7.0 & 0.604 \\
\hline & st. deviation & 3.6 & 0.0049 & 4.6 & 0.0151 & 2.4 & 0.0241 \\
\hline \multirow{2}{*}{2500} & average & 37.5 & 0.828 & 55.4 & 0.740 & 6.3 & 0.600 \\
\hline & st. deviation & 3.0 & 0.0045 & 3.9 & 0.0133 & 1.5 & 0.0225 \\
\hline
\end{tabular}


Table 4: Mean machine settings parameters for different sample sizes

\begin{tabular}{|c|l|r|r|r|}
\hline \multirow{2}{*}{$\begin{array}{c}\text { Sample } \\
\text { size }\end{array}$} & \multirow{3}{*}{} & \multicolumn{3}{|c|}{ Independent sampling } \\
\cline { 3 - 5 } & & \multicolumn{3}{|c|}{ Grades } \\
\cline { 3 - 5 } 250 & average & 9944 & 8212 & 6767 \\
\cline { 2 - 5 } & st. deviation & 317 & 404 & 594 \\
\hline \multirow{2}{*}{500} & average & 9905 & 7887 & 6479 \\
\cline { 2 - 5 } & st. deviation & 206 & 260 & 263 \\
\hline \multirow{2}{*}{750} & average & 9885 & 7821 & 6468 \\
\cline { 2 - 5 } & st. deviation & 169 & 224 & 299 \\
\hline
\end{tabular}

\begin{tabular}{|c|l|r|r|r|}
\hline \multirow{2}{*}{$\begin{array}{c}\text { Sample } \\
\text { size }\end{array}$} & & \multicolumn{3}{|c|}{ Sampling from finite population } \\
\cline { 3 - 5 } & & \multicolumn{3}{|c|}{ Grades } \\
\cline { 3 - 5 } & & C40 & \multicolumn{1}{c|}{ C30 } & \multicolumn{1}{c|}{ C18 } \\
\hline \multirow{2}{*}{250} & average & 9958 & 8225 & 6848 \\
\cline { 2 - 5 } & st. deviation & 259 & 369 & 520 \\
\hline \multirow{2}{*}{500} & average & 9907 & 7929 & 6663 \\
\cline { 2 - 5 } & st. deviation & 124 & 233 & 291 \\
\hline \multirow{2}{*}{750} & average & 9931 & 7866 & 6592 \\
\cline { 2 - 5 } & st. deviation & 85 & 186 & 170 \\
\hline
\end{tabular}


Table 5: Mean machine settings parameters for three different populations of 1000 pieces

\begin{tabular}{|c|l|l|r|r|r|}
\hline \multirow{2}{*}{ No. } & \multirow{2}{*}{$\begin{array}{l}\text { Sample } \\
\text { size }\end{array}$} & & \multicolumn{3}{|c|}{ Sampling from finite population } \\
\cline { 4 - 6 } & & & \multicolumn{3}{|c|}{ Grades } \\
\hline \multirow{2}{*}{1} & \multirow{2}{*}{250} & average & 9958 & 8223 & 6809 \\
\cline { 3 - 6 } & & st. deviation & 263 & 360 & 503 \\
\hline \multirow{2}{*}{2} & \multirow{2}{*}{250} & average & 9772 & 8459 & 7138 \\
\cline { 3 - 6 } & & st. deviation & 190 & 479 & 806 \\
\hline \multirow{2}{*}{3} & 250 & average & 10007 & 8232 & 6959 \\
\cline { 3 - 6 } & & st. deviation & 306 & 428 & 637 \\
\hline
\end{tabular}


Table 6: Confidence intervals for yield to different strength classes [\%]. It is assumed that independent sampling is repeated 4 times.

\begin{tabular}{|c|c|c|c|c|c|c|}
\hline \multirow{2}{*}{$\begin{array}{l}\text { Sample } \\
\text { size }\end{array}$} & \multicolumn{6}{|c|}{ Yield in grades $[\%]$} \\
\hline & \multicolumn{2}{|c|}{ C40 } & \multicolumn{2}{|c|}{ C30 } & \multicolumn{2}{|c|}{ C18 } \\
\hline \multirow{2}{*}{250} & \multicolumn{2}{|c|}{$35.3 \pm 9.3 \cdot 1.96 / \sqrt{4}$} & \multicolumn{2}{|c|}{$48.6 \pm 14.1 \cdot 1.96 / \sqrt{4}$} & \multicolumn{2}{|c|}{$13.6 \pm 7.9 \cdot 1.96 / \sqrt{4}$} \\
\hline & 26.2 & 44.4 & 34.8 & 62.4 & 5.9 & 21.3 \\
\hline \multirow{2}{*}{500} & \multicolumn{2}{|c|}{$35.9 \pm 6.1 \cdot 1.96 / \sqrt{4}$} & \multicolumn{2}{|c|}{$50.8 \pm 9.7 \cdot 1.96 / \sqrt{4}$} & \multicolumn{2}{|c|}{$11.0 \pm 6.4 \cdot 1.96 / \sqrt{4}$} \\
\hline & 30.0 & 41.8 & 41.3 & 60.3 & 4.7 & 17.3 \\
\hline \multirow{2}{*}{750} & \multicolumn{2}{|c|}{$36.7 \pm 5.4 \cdot 1.96 / \sqrt{4}$} & \multicolumn{2}{|c|}{$54.4 \pm 7.1 \cdot 1.96 / \sqrt{4}$} & \multicolumn{2}{|c|}{$8.1 \pm 3.1 \cdot 1.96 / \sqrt{4}$} \\
\hline & 31.4 & 42.0 & 47.5 & 61.3 & 5.1 & 11.1 \\
\hline \multirow{2}{*}{1000} & \multicolumn{2}{|c|}{$37.1 \pm 5.0 \cdot 1.96 / \sqrt{4}$} & \multicolumn{2}{|c|}{$52.3 \pm 7.3 \cdot 1.96 / \sqrt{4}$} & \multicolumn{2}{|c|}{$8.4 \pm 4.8 \cdot 1.96 / \sqrt{4}$} \\
\hline & 32.2 & 42.0 & 45.1 & 59.5 & 3.7 & 13.0 \\
\hline \multirow{2}{*}{1250} & \multicolumn{2}{|c|}{$36.4 \pm 3.6 \cdot 1.96 / \sqrt{4}$} & \multicolumn{2}{|c|}{$55.9 \pm 4.5 \cdot 1.96 / \sqrt{4}$} & \multicolumn{2}{|c|}{$7.0 \pm 2.4 \cdot 1.96 / \sqrt{4}$} \\
\hline & 32.8 & 40.0 & 51.5 & 60.3 & 3.7 & 13.0 \\
\hline \multirow{2}{*}{2500} & \multicolumn{2}{|c|}{$37.5 \pm 3.0 \cdot 1.96 / \sqrt{4}$} & \multicolumn{2}{|c|}{$55.4 \pm 3.9 \cdot 1.96 / \sqrt{4}$} & \multicolumn{2}{|c|}{$6.4 \pm 1.6 \cdot 1.96 / \sqrt{4}$} \\
\hline & 34.6 & 40.4 & 51.6 & 59.2 & 4.8 & 7.9 \\
\hline
\end{tabular}




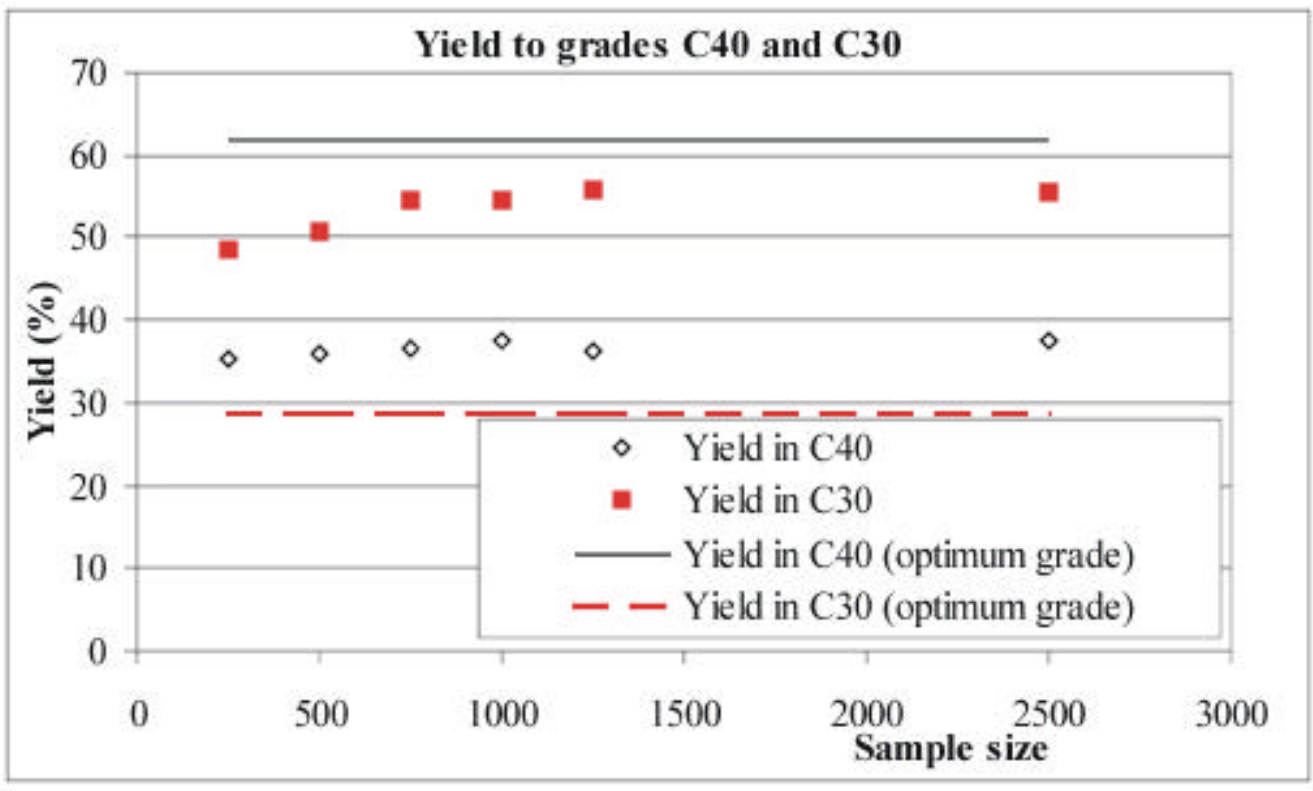

Figure 1: Average yield in C40 and C30 for the generated samples of spruce with $150 \mathrm{~mm}$ depth 


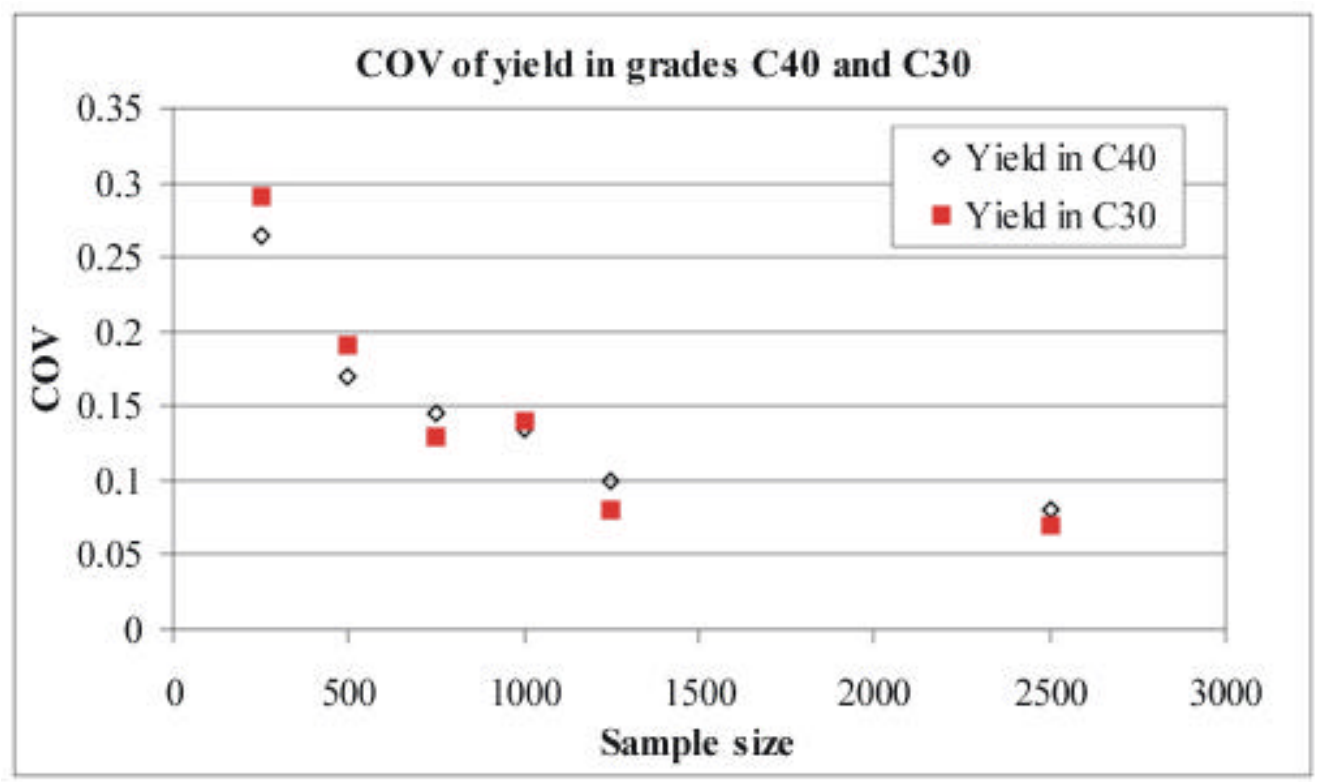

Figure 2: Coefficient of variation for yield in C40 and C30 for the generated samples of spruce with $150 \mathrm{~mm}$ depth 


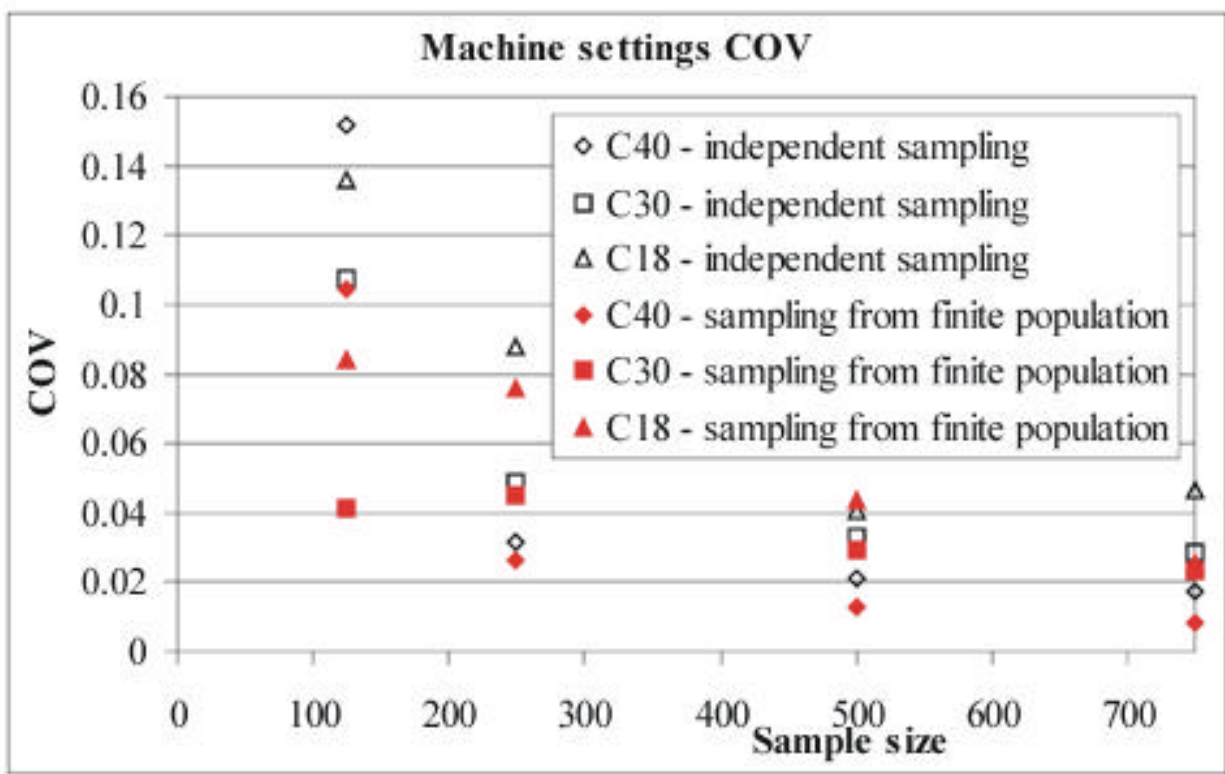

Figure 3: Coefficients of variation of machine settings for the cases of independent sampling and sampling from the finite population of 1000 pieces 


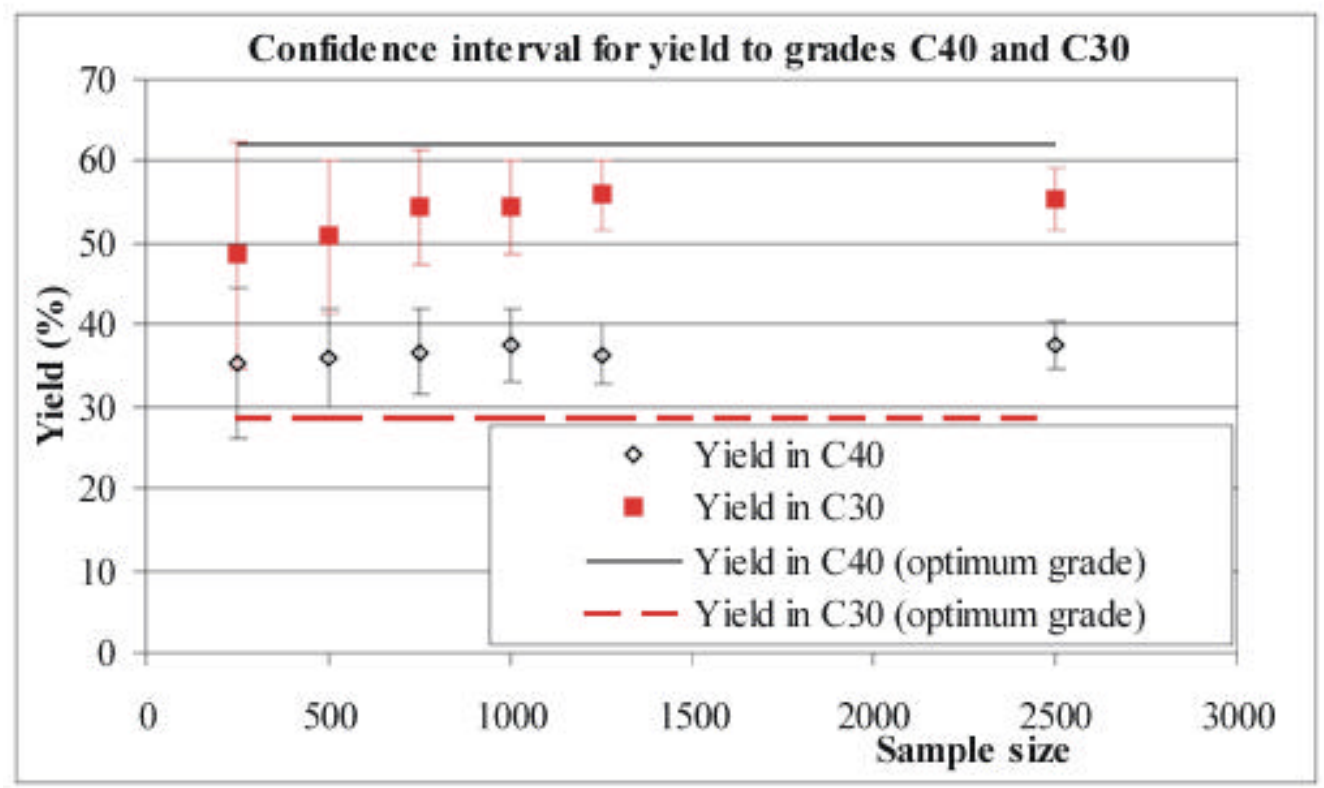

Figure 4: Yield in C40 and C30 - $95 \%$ confidence intervals (4 repetitions of sampling) 


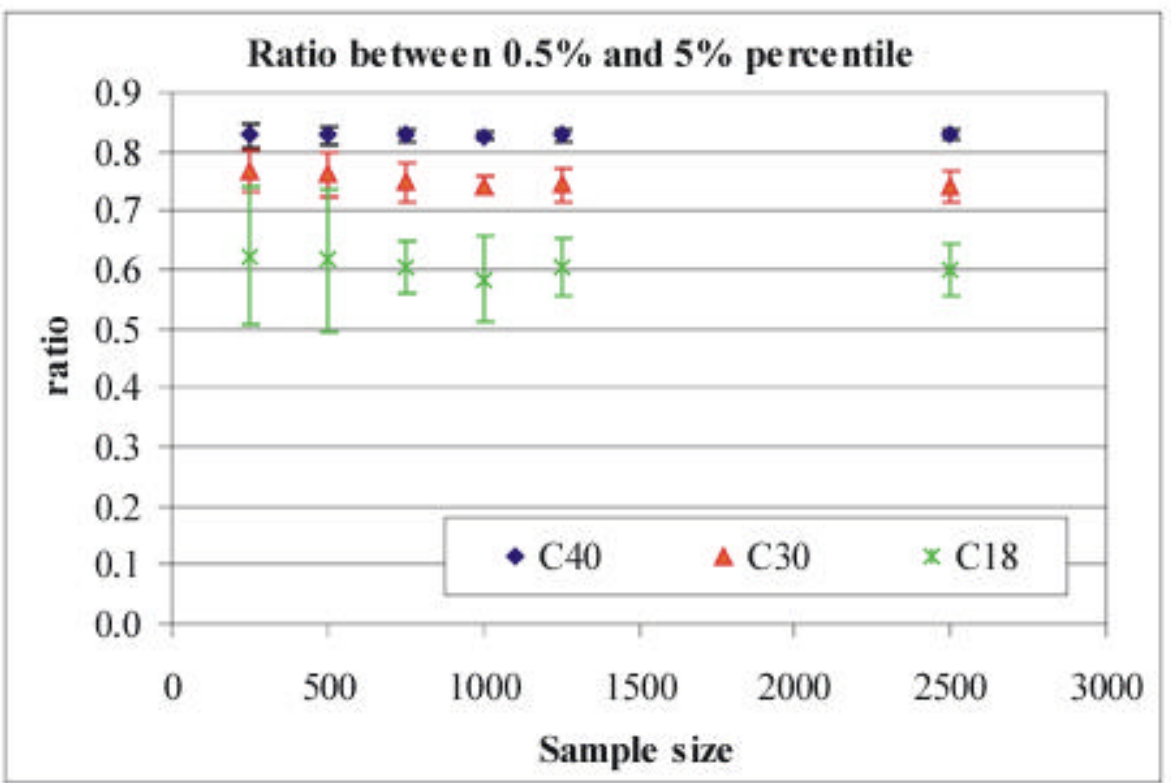

Figure 5: Ratio between $0.5 \%$ and $5 \%$ percentile of the strength 


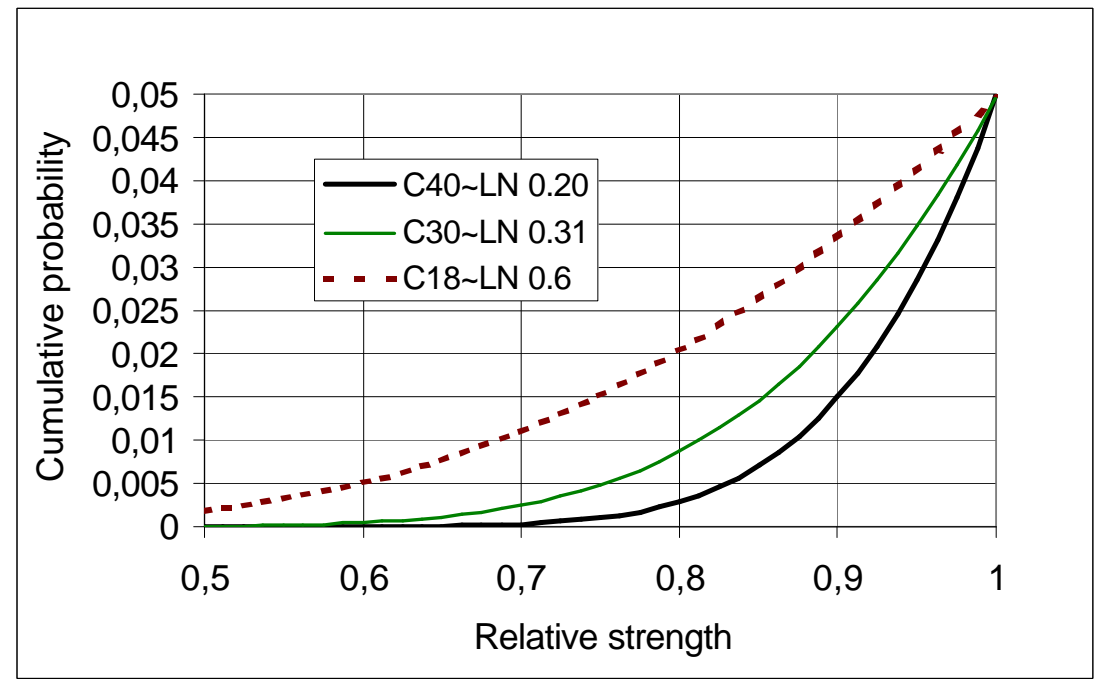

Figure 6: Relative strength of timber graded simultaneously to C40, C30 and C18 